\title{
The circum-galactic medium of quasars: transverse and line-of-sight absorptions
}

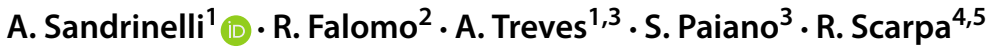

Received: 9 September 2020 / Accepted: 31 October 2020 / Published online: 12 November 2020

(c) The Author(s) 2020

\begin{abstract}
Quasar projected pairs (QPPs) can be used for investigating the circumgalactic medium of quasars through the study of intervening absorption lines in the spectrum of the background quasar $\left(\mathrm{QSO}_{\mathrm{B}}\right)$ that are at the same redshift of the foreground quasar $\left(\mathrm{QSO}_{\mathrm{F}}\right)$. Here we report on optical spectroscopy, gathered at Gran Telescopio Canarias, of 14 QPPs. In 7 cases we find MgII absorption lines associated with the foreground quasar. Only for two cases line-ofsight absorptions (LOS) are revealed. These new observations complement our previous study performed on other 30 QPPs. A brief discussion of the properties of the intervening absorption lines associated with the foreground quasar for the full dataset is reported.
\end{abstract}

Keywords Galaxies: active · Galaxies: haloes - Quasars: absorption lines · Quasars: general

\section{Introduction}

The circum-galactic medium (CGM) is the diffuse gas spanning a few hundred kpc outside the galaxies and represents

$\otimes$ A. Sandrinelli

asandrinelli@yahoo.it

1 Istituto Nazionale di Astrofisica - Osservatorio Astronomico di Brera, INAF, via E. Bianchi 46, 23807 Merate, LC, Italy

2 Istituto Nazionale di Astrofisica - Osservatorio Astronomico di Padova, INAF, Vicolo dell'Osservatorio 5, 35122 Padova, PD, Italy

3 Dipartimento di Scienza e Alta Tecnologia (DISAT), Università degli Studi dell'Insubria, via Valleggio 11, 22100 Como, Italy

4 Instituto de Astrofısica de Canarias, c/via Lactea s/n San Cristobal de la Laguna, 38205, Spain

5 Departamento de Astrofisica, Universidad de La Laguna (ULL), 38206 La Laguna, Tenerife, Spain the most abundant reservoir of baryons of galaxies (e.g. Peek et al. 2015; Telford et al. 2019). It is the link between the interstellar and the intergalactic media, so that gas exchanges between them have to go through it and necessarily leave here the proof of its passage (see e.g. Tumlinson et al. 2017, for a review). These exchanges play an important role in the evolution of a galaxy because they can favour or suppress different processes in the galaxy, notably star formation. Various feedback mechanisms are invoked in hydrodynamic simulations for discriminating between possible theoretical models (e.g. Oppenheimer et al. 2010; Fielding et al. 2017).

An effective method for probing the properties of the CGM of a galaxy is to study the absorption spectrum of a background source which is angularly close so that the projected distance $\left(R_{\perp}\right)$ is of the order of hundreds kpc. Quasars are sources apt to this scope since they are bright and point-like. If one is interested in the CGM of a quasar, the system is a quasar projected pair (QPP): the background quasar $\left(\mathrm{QSO}_{\mathrm{B}}\right)$ is the light source, which enables to explore the GCM of the foreground quasar $\left(\mathrm{QSO}_{\mathrm{F}}\right)$ at different projected distance, or impact parameter, from the $\mathrm{QSO}_{\mathrm{F}}$. This approach was introduced by Hennawi et al. (2006), who studied the Ly $\alpha$ absorption systems in the halo of quasars at $\mathrm{z} \sim 2$ and found a high fraction of absorbers for small separations $\left(\mathrm{R}_{\perp}<150 \mathrm{~h}^{-1} \mathrm{kpc}\right)$ coincident with the foreground quasar. This provided significant evidence that these absorbers are strongly clustered around quasars. Using $10 \mathrm{~m}$ class telescopes we investigated the presence of intervening MgII absorption systems in the CGM of QSOs at $\mathrm{z} \sim 1$ (Farina et al. 2013, 2014) by examining 30 QPPs with angular separations $10^{\prime \prime}-30^{\prime \prime}$ in order to explore the CGM in the 50-200 kpc region. In about half cases MgII absorptions due to the foreground quasar were detected in the background quasar spectrum (traverse absorptions). The indica- 
tion is that the covering of the gas drops quickly beyond 100 kpc. In only one case MgII absorption along the line-of-sight (LOS) was revealed.

These results were also confirmed by Johnson et al. (2015) who carried out a study of a data set of QPPs from SDSS DR12 spectral archives. They found a strong dependence of the absorption equivalent width on the luminosity/mass of the $\mathrm{QSO}_{\mathrm{F}}$, point which was already suggested in Farina et al. (2014).

In this paper we report observations of 14 new QPPs obtained at the $10.4 \mathrm{~m}$ Gran Telescopio Canarias (GTC) (Sect. 2). Our main aim is to enlarge our previous sample and in compare the transverse and LOS MgII absorption lines. For this work we assume the following cosmology: $\mathrm{H}_{0}=70 \mathrm{~km} \mathrm{~s}^{-1} \mathrm{Mpc}^{-1}, \Omega_{\mathrm{m}}=0.3$ and $\Omega_{\Lambda}=0.7$.

\section{Properties of the projected quasar pairs}

In order to extend the data set of our previous work (Farina et al. 2013,2014$)$ towards small $\left(\mathrm{R}_{\perp} \lesssim 50 \mathrm{kpc}\right)$ and large $\left(\mathrm{R}_{\perp} \gtrsim 200 \mathrm{kpc}\right)$ projected distances, we searched for new targets in The Million Quasars Catalog (Flesch 2015, 2019), following the selection criteria given below. We required projected distance of the pairs to be less than 250 $\mathrm{kpc}$ at the redshift of foreground quasar, which allows us to explore the outer regions of a CGM halo. Differences between the radial velocities of the two sources were chosen

Table 1 The sample of quasar projected pairs and properties. In columns: identification label of the system (ID), position of the foreground target, redshift derived from literature (z), V-band apparent and absolute magnitude, angular $(\Delta \theta)$ and projected $\left(\mathrm{R}_{\perp}\right)$ separation be-
$>5000 \mathrm{~km} / \mathrm{s}$ to ensure the quasar pair is not a gravitational bound system. The redshifts are drawn from SDSS archives, when present, otherwise from literature.

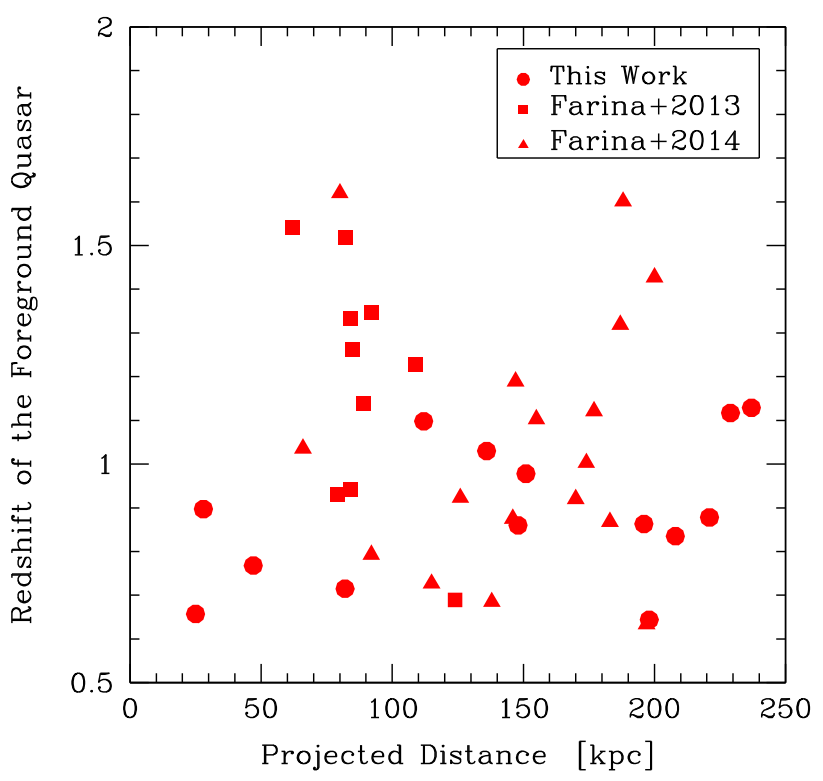

Fig. 1 Distribution in the $\mathrm{R}_{\perp}-\mathrm{Z}_{F}$ plane of the projected QSO pairs studied to investigate the MgII absorbing circumgalactic medium of quasars. Triangles and squares are objects investigated in Farina et al. (2013) and Farina et al. (2014), respectively. Newly observed targets from this work are reported as circles

tween the two quasars, seeing during the observations (see), signalto-noise ratio $(\mathrm{SN})$ of the spectrum. The labels $\mathrm{F}$ and $\mathrm{B}$ refer to the foreground and background quasar, respectively

\begin{tabular}{|c|c|c|c|c|c|c|c|c|c|c|c|c|}
\hline ID & $\begin{array}{l}\mathrm{QSO}_{\mathrm{F}} \text { coordinates } \\
(\mathrm{J} 2000)\end{array}$ & $\mathrm{zF}_{\mathrm{F}}$ & $\mathrm{z}_{\mathrm{B}}$ & $\begin{array}{l}\mathrm{V}_{\mathrm{F}} \\
(\mathrm{mag})\end{array}$ & $\begin{array}{l}\mathrm{V}_{\mathrm{B}} \\
(\mathrm{mag})\end{array}$ & $\begin{array}{l}\mathrm{M}(\mathrm{V})_{\mathrm{F}} \\
(\mathrm{mag})\end{array}$ & $\begin{array}{l}\mathrm{M}(\mathrm{V})_{\mathrm{B}} \\
(\mathrm{mag})\end{array}$ & $\begin{array}{l}\Delta \theta \\
(\operatorname{arcsec})\end{array}$ & $\begin{array}{l}\mathrm{R}_{\perp} \\
(\mathrm{kpc})\end{array}$ & $\begin{array}{l}\text { see } \\
(\operatorname{arcsec})\end{array}$ & $\mathrm{SN}_{\mathrm{F}}$ & $\mathrm{SN}_{\mathrm{B}}$ \\
\hline QQ01 & $08: 36: 49.47+48: 41: 50.1$ & 0.657 & 1.711 & 19.14 & 18.66 & -23.71 & -26.59 & 3.7 & 25 & 0.8 & 20 & 30 \\
\hline QQ02 & $08: 59: 15.10+42: 41: 23.6$ & 0.901 & 1.396 & 19.37 & 20.79 & -24.18 & -23.96 & 3.6 & 30 & 0.8 & 30 & 10 \\
\hline QQ03 & $09: 28: 27.92-00: 11: 26.5$ & 0.878 & 1.138 & 19.24 & 20.24 & -24.16 & -23.91 & 28.6 & 221 & 1.6 & 10 & 10 \\
\hline QQ04 & $10: 19: 57.47-02: 43: 05.5$ & 0.863 & 0.993 & 20.58 & 20.20 & -23.29 & -23.61 & 25.4 & 196 & 1.3 & 10 & 15 \\
\hline QQ05 & $10: 27: 53.84+00: 30: 55.4$ & 1.129 & 1.904 & 18.63 & 21.30 & -25.50 & -24.77 & 28.9 & 237 & 1.9 & 95 & 10 \\
\hline QQ06 & $10: 44: 31.74+61: 38: 48.4$ & 1.096 & 2.522 & 20.07 & 20.38 & -23.98 & -25.99 & 13.7 & 112 & 0.8 & 15 & 15 \\
\hline QQ07 & $11: 14: 43.15-00: 51: 21.5$ & 0.835 & 1.815 & 20.26 & 20.70 & -23.52 & -25.24 & 27.3 & 208 & 1.3 & 10 & 20 \\
\hline QQ08 & $13: 33: 16.10+00: 36: 24.9$ & 1.030 & 1.794 & 20.98 & 20.58 & -22.92 & -24.80 & 16.9 & 136 & 2.5 & 6 & 10 \\
\hline QQ09 & $13: 39: 45.07+00: 10: 04.5$ & 0.978 & 1.873 & 20.02 & 19.23 & -23.75 & -26.29 & 19.0 & 151 & 2.4 & 10 & 23 \\
\hline QQ10 & $15: 45: 43.05+05: 49: 29.0$ & 0.644 & 1.226 & 19.71 & 18.99 & -23.09 & -25.37 & 28.7 & 198 & 1.8 & 20 & 30 \\
\hline QQ11 & $17: 11: 29.32+29: 15: 23.0$ & 1.117 & 2.163 & 20.32 & 20.52 & -23.78 & -25.42 & 27.9 & 229 & 0.8 & 15 & 10 \\
\hline QQ12 & $21: 20: 27.06-00: 19: 51.1$ & 0.86 & 2.578 & 20.69 & 17.77 & -22.76 & -28.67 & 19.3 & 148 & 0.7 & 10 & 45 \\
\hline QQ13 & $23: 12: 52.80+14: 44: 58.6$ & 0.768 & 1.523 & 19.93 & 17.74 & -23.28 & -27.24 & 6.3 & 47 & 0.8 & 10 & 105 \\
\hline QQ14 & $23: 13: 03.84+10: 49: 15.5$ & 0.713 & 1.333 & 19.70 & 18.90 & -23.35 & -25.71 & 11.3 & 82 & 1.8 & 20 & 20 \\
\hline
\end{tabular}



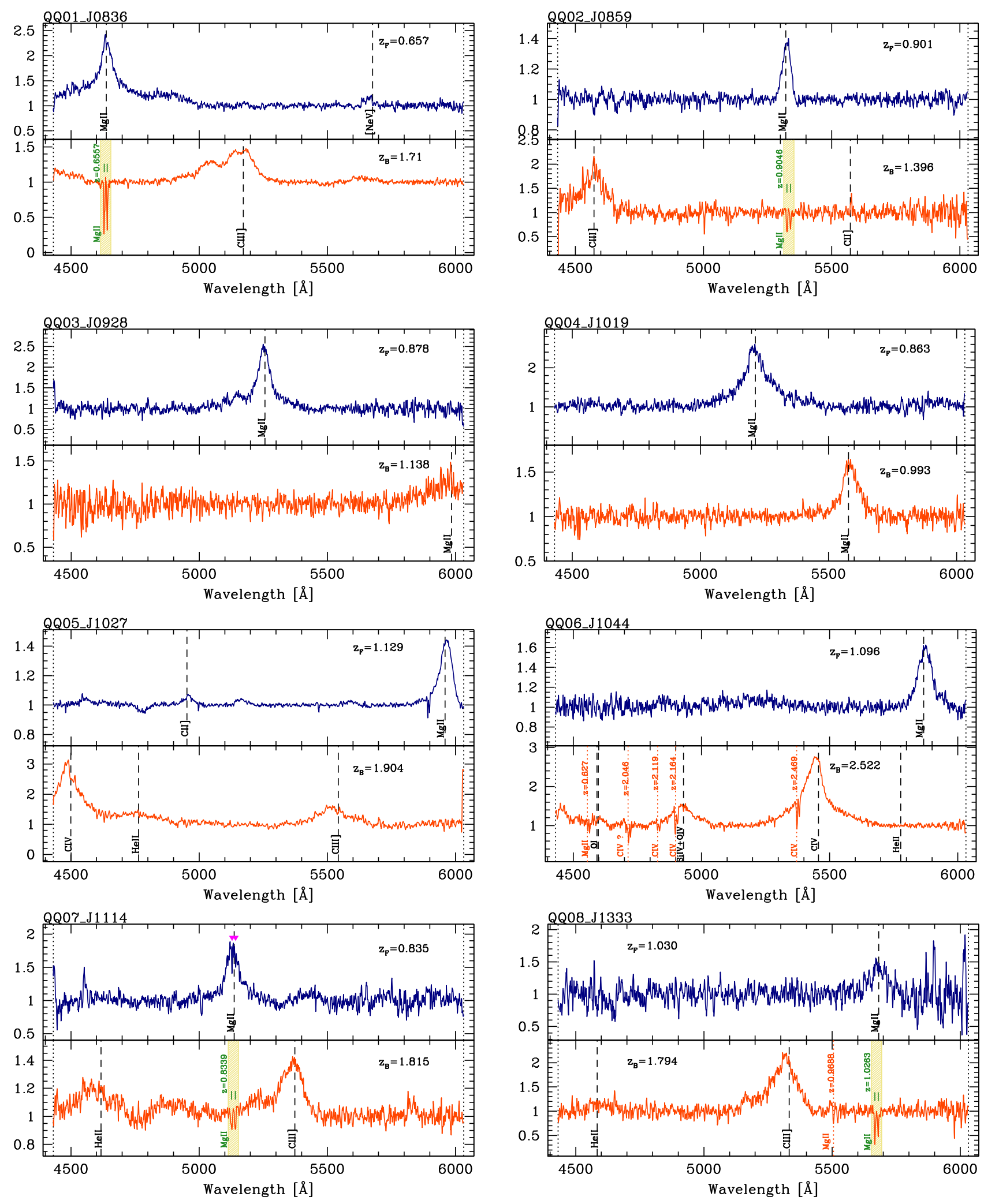

Fig. 2 Normalized QSOs spectra. The spectra of the $\mathrm{QSO}_{\mathrm{F}}$ and the $\mathrm{QSO}_{\mathrm{B}}$ are displayed at the top and at the bottom panel of each frame, respectively. The main emission lines are labeled and unidentified by dashed black lines. Detected $\mathrm{MgII}$ absorption features in the $\mathrm{QSO}_{\mathrm{B}}$ spectrum associated with the $\mathrm{QSO}_{\mathrm{F}}$ are evidenced by a yellow bar, see

also Fig. 3, the redshift of the absorbers are reported in green. The positions of line-of-sight (LOS) absorbers in the foreground QSO spectra are singled out by magenta triangles, see also Fig. 4. Red dotted lines mark absorption systems due to intervening clouds or transverse absorption features of CIV 

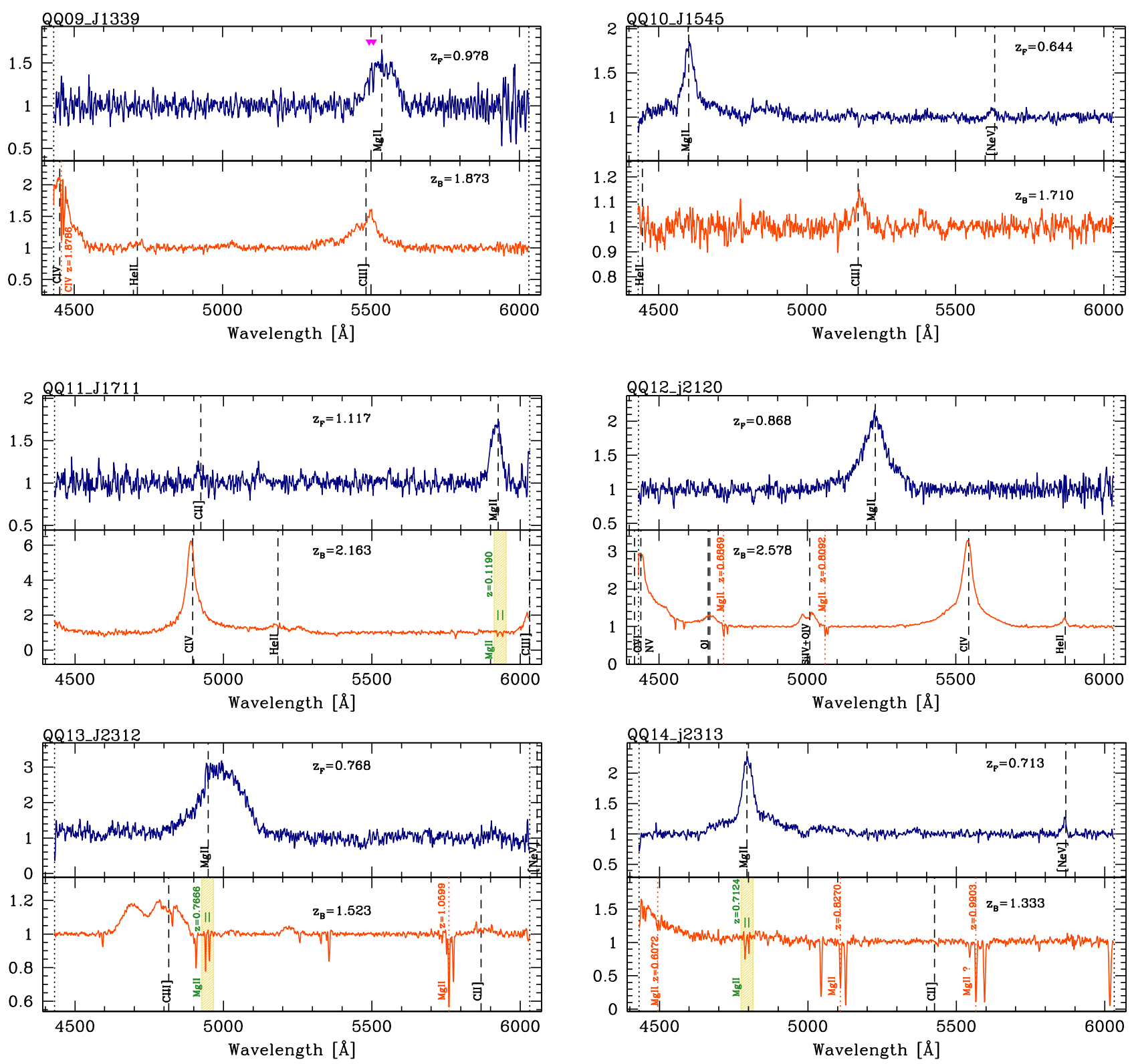

Fig. 2 (Continued)

The MgII doublet absorption systems in the background quasar spectrum, associated to the $\mathrm{MgII}$ emission in the foreground quasar, have to fall inside the spectral range (4500$6000 \AA$ ) of the adopted grism (see Sect. 3) constraining the foreground quasars at redshift interval $0.6<\mathrm{z}<1.15$. Paired targets are also selected ensuring good visibility from the GTC site in Roque de los Muchachos, La Palma, and request that the background quasar be brighter than $\mathrm{V}=20.8$ in order to obtain spectra with signal-to-noise ratio $\gtrsim 10$ in normal weather conditions. From this new selection 14 QQPs have been targeted at the GTC, yielding the full data set given in Fig. 1 in the $R_{\perp}$-z plane. In Table 1 we report the general properties of the newly observed pairs.

\section{Observations and data reduction}

The QPP spectra were secured at the GTC from Sept. 2017 to Aug. 2018 equipped with the Optical System for Imaging and Low Resolution Integrated Spectroscopy (OSIRIS, Cepa et al. 2003) R2500V grism, yielding effective intermediate spectral resolution $\mathrm{R}=1500$ for a slit of 1.00 ”, with 1 px corresponding to $1.3 \AA$ or to $\sim 70 \mathrm{~km} \mathrm{~s}^{-1}$ at the central spectral wavelength. This allows us to fully resolve the MgII doublet components $(\lambda 2797 \AA$ and $\lambda 2803 \AA$ ). The spectral window covers the 4500-6000 $\AA$ range. For each QPP the slit was oriented to simultaneously gather the spectrum of both objects. The full observation of each QSO pair was divided into three exposures $(3 \times 1200 \mathrm{~s})$ in order to provide 
Table 2 Properties of MgII transverse absorption features in the spectrum of the background QSO and associated to the foreground QSO. The columns show the observed wavelengths $\left(\lambda_{\text {abs }}\right)$, the rest-frame equivalent width $\left(\mathrm{EW}_{\text {rest }}\right)$, the redshift of the absorber $\left(\mathrm{z}_{\mathrm{abs}}\right)$. The $2 \sigma$ upper limit for the minimum equivalent width $\mathrm{EW}_{\text {rest }}$ detectable in the background QSO spectrum is also quoted

\begin{tabular}{|c|c|c|c|c|c|c|}
\hline ID & $\begin{array}{l}\lambda_{\text {abs }}(\lambda 2796) \\
(\AA)\end{array}$ & $\begin{array}{l}\mathrm{EW}_{\text {rest }}(\lambda 2796) \\
(\AA)\end{array}$ & $\begin{array}{l}\lambda_{\mathrm{abs}}(\lambda 2803) \\
(\AA)\end{array}$ & $\begin{array}{l}\mathrm{EW}_{\text {rest }}(\lambda 2803) \\
(\AA)\end{array}$ & $\mathrm{z}_{\mathrm{abs}}$ & $\begin{array}{l}\mathrm{EW}_{\text {min,rest }} \\
(\AA)\end{array}$ \\
\hline QQ01 & 4629.8 & $1.84 \pm 0.10$ & 4641.0 & $1.56 \pm 0.08$ & 0.6556 & 0.13 \\
\hline QQ02 & 5326.0 & $1.17 \pm 0.18$ & 5339.3 & $1.57 \pm 0.39$ & 0.9046 & 0.38 \\
\hline QQ03 & - & - & - & - & - & 0.27 \\
\hline QQ04 & - & - & - & - & - & 0.42 \\
\hline QQ05 & - & - & - & - & - & 0.36 \\
\hline QQ06 & - & - & - & - & - & 0.19 \\
\hline QQ07 & 5126.6 & $0.51 \pm 0.24$ & 5141.6 & $0.44 \pm 0.19$ & 0.8340 & 0.19 \\
\hline QQ08 & 5667.0 & $1.64 \pm 0.15$ & 5679.8 & $1.18 \pm 0.21$ & 1.0263 & 0.76 \\
\hline QQ09 & - & - & - & - & - & 0.20 \\
\hline QQ10 & - & - & - & - & - & 0.19 \\
\hline QQ11 & 5924.4 & $0.51 \pm 0.15$ & 5940.7 & $0.51 \pm 0.27$ & 1.1190 & 0.49 \\
\hline QQ12 & - & - & - & - & - & 0.11 \\
\hline QQ13 & 4940.0 & $0.36 \pm 0.05$ & 4952.7 & $0.21 \pm 0.06$ & 0.7666 & 0.06 \\
\hline QQ14 & 4788.4 & $0.71 \pm 0.16$ & 4801.2 & $0.44 \pm 0.18$ & 0.7125 & 0.29 \\
\hline
\end{tabular}
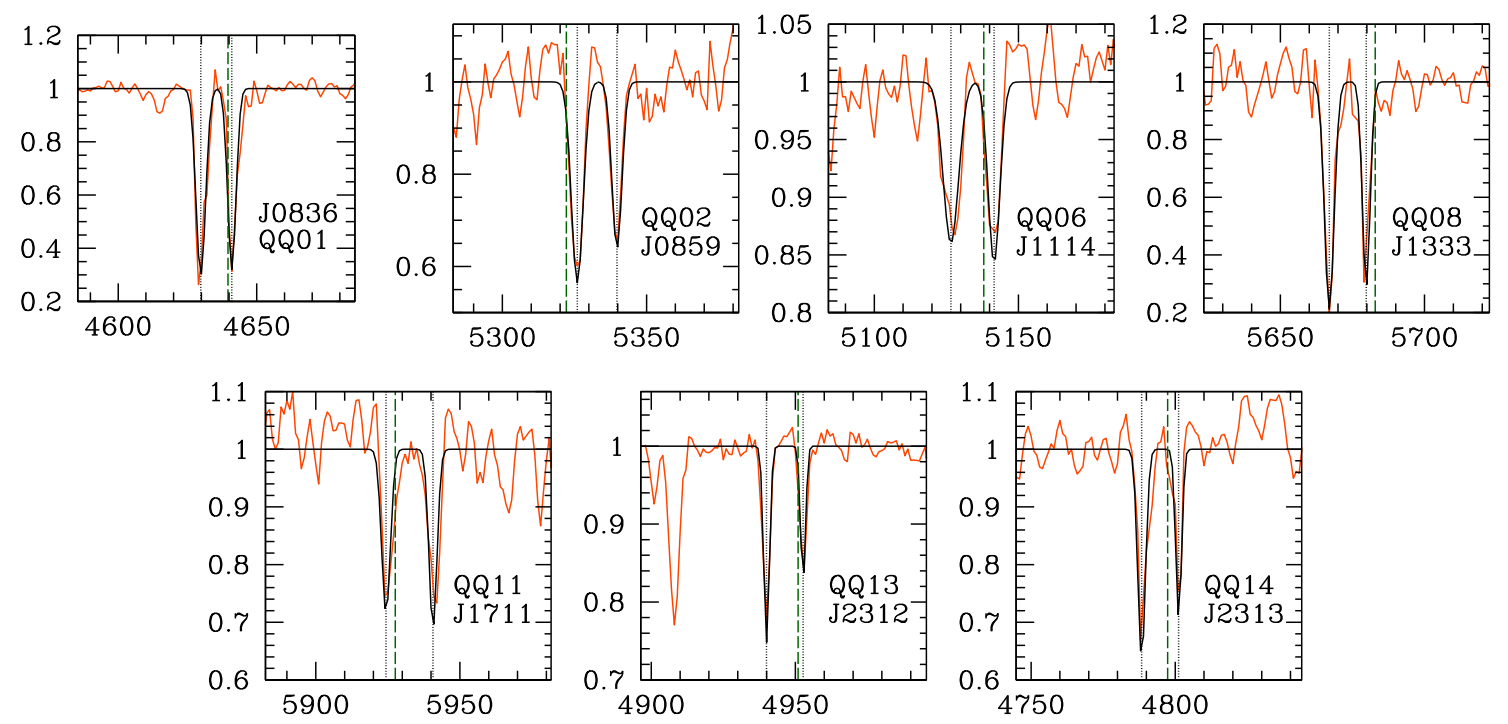

Fig. 3 Close-ups of the normalized $\mathrm{QSO}_{\mathrm{B}}$ spectra (red lines) presenting transverse absorption systems associated to the $\mathrm{MgII}$ emission line of the $\mathrm{QSO}_{\mathrm{F}}$. Gaussian fits are performed on the absorptions lines and drawn as solid black lines. Dotted black lines indicate the positions of spectral absorption peaks, while the green dashed line marks the position of the MgII emission line in the $\mathrm{QSO}_{\mathrm{F}}$ spectrum

tions have been introduced through aperture photometry of the field acquired concurrently to the spectra.

The GTC spectra, normalised to the continuum level, are showed in Fig. 2.

\section{Transverse absorption systems}

We search for $\operatorname{MgII}(\lambda 2797, \lambda 2803)$ absorption features with $\mathrm{EW}_{\text {rest }}(\lambda 2796)$ larger than the minimum observable equiv- 
Table 3 Properties of MgII line-of-sight absorption systems in the foreground QSOs. The radial velocity difference $\Delta \mathrm{V}$ between the absorptionsystem and the foreground QSO is added

\begin{tabular}{|c|c|c|c|c|c|c|c|}
\hline ID & $\begin{array}{l}\lambda_{\text {abs }}(\lambda 2796) \\
(\AA)\end{array}$ & $\begin{array}{l}\mathrm{EW}_{\text {rest }}(\lambda 2796) \\
(\AA)\end{array}$ & $\begin{array}{l}\lambda_{\text {abs }}(\lambda 2803) \\
(\AA)\end{array}$ & $\begin{array}{l}\mathrm{EW}_{\text {rest }}(\lambda 2803) \\
(\mathrm{km} / \mathrm{s})\end{array}$ & $\begin{array}{l}Z_{\text {abs }} \\
(\AA)\end{array}$ & $\Delta \mathrm{V}$ & $\mathrm{EW}_{\text {min, rest }}$ \\
\hline QQ07 & 5128.6 & $0.43 \pm 0.11$ & 5142.2 & $0.25 \pm 0.15$ & 0.8341 & -250 & 0.35 \\
\hline QQ09 & 5493.9 & $0.35 \pm 0.10$ & 5508.3 & $0.46 \pm 0.14$ & 0.9647 & -2000 & 0.32 \\
\hline
\end{tabular}
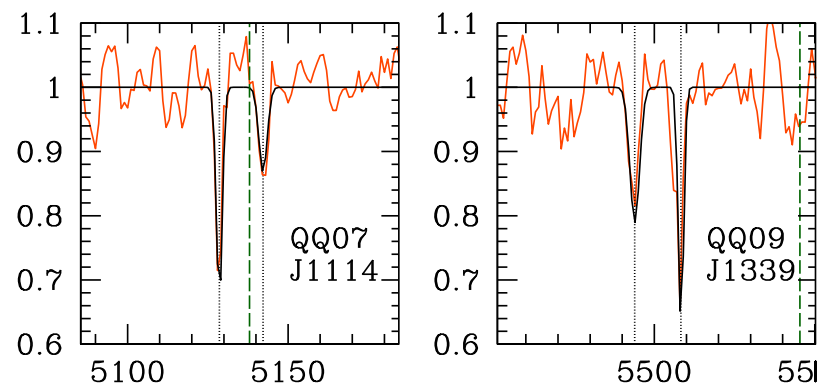

Fig. 4 Close-ups of the normalized QSOF spectra (red lines) presenting line-of-sight absorption systems associated to the MgII emission line of the $\mathrm{QSO}_{\mathrm{F}}$ itself. The spectra are normalised along the MgII emission line profile. Gaussian fits are performed on the absorptions lines and drawn as solid black lines. Dotted black lines indicate the positions of spectral absorption peaks, while the green dashed line marks the position of the $\mathrm{MgII}$ emission line in the $\mathrm{QSO}_{\mathrm{F}}$ spectrum

alent width $\left(\mathrm{EW}_{\min }\right.$, see Paiano et al. 2017) in the $\mathrm{QSO}_{\mathrm{B}}$ spectrum.

We detect transverse MgII absorptions in 7 pairs, see Fig. 2 and Table 2. Close-ups of the absorptions with gaussian fits are reported in Fig. 3. The covering fraction $f_{C}(0.3 \AA)$, i.e. the fraction of cases where MgII absorptions are apparent with a sensitivity threshold corresponding to a rest-frame equivalent width $\mathrm{EW}_{\text {rest }}(\lambda 2796) \gtrsim 0.3 \AA$, in our new sample is $50 \%$.

We explore the extent and the properties of the MgII lowdensity absorbing gas located in the CGM environment of quasars using the total sample of 44 projected pairs located at $0.6 \lesssim \mathrm{z}_{F} \lesssim 2.2$ and investigated by our team in this work and in Farina et al. $(2013,2014)$. Over the whole range of projected distances $\left(\sim 30 \mathrm{kpc}<\mathrm{R}_{\perp}<\sim 250 \mathrm{kpc}\right)$ we find $f_{C}(0.3 \AA)=0.45$ and $f_{C}(0.6 \AA)=0.25$. In Fig. 5, we show the MgII covering fraction profile of transverse absorption systems with $\mathrm{EW}_{\text {rest }}(2796)>0.3 \AA$ against the impact parameter, where binomial $1 \sigma$ confidence intervals are taken as uncertainties (Gehrels 1986). The covering fraction is $f c(0.3 \AA)=1.00_{0.69}^{0.00}$ in the first bin $\left(20 \mathrm{kpc}<\mathrm{R}_{\perp}<75 \mathrm{kpc}\right)$ and decreases with the impact parameter. Note however that we observe the presence of three absorbers over $200 \mathrm{kpc}$, and this yields $f c(0.3 \AA)=0.30_{0.14}^{0.51}$ in the range $185 \mathrm{kpc}<$ $\mathrm{R}_{\perp}<240 \mathrm{kpc}$.

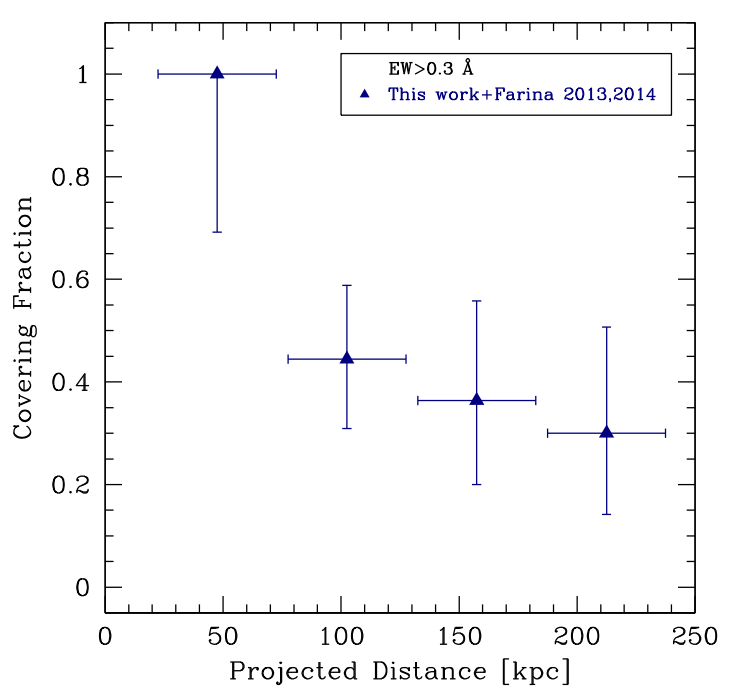

Fig. 5 Covering fraction profile for transverse absorption systems in the $\mathrm{QSO}_{B}$ spectrum associated with the $\mathrm{QSO}_{F}$ with $\mathrm{EW}(2796)>0.3 \AA$, plotted as a function of projected distance between the two QSOs. Triangles refer to absorption systems investigated in our works, this and Farina et al. 2013, 2014. Horizontal bars represent the impact parameter of projected distance, which span from 20 to 240 $\mathrm{kpc}$ in $55 \mathrm{kpc}$ wide intervals. In each bin the $1 \sigma$ binomial confidence intervals is reported as vertical error bar (Gehrels 1986)

\section{Line of sight absorptions}

We now focus on LOS absorptions, which are absorption features superposed to the MgII emission line of the $\mathrm{QSO}_{\mathrm{F}}$. For attributing the absorption to the CGM cloud Farina et al. (2014) considered two possible velocity difference thresholds $1000 \mathrm{~km} / \mathrm{s}$, or following Sharma et al. (2013), $5000 \mathrm{~km} / \mathrm{s}$.

Taking the former value, we find that within our sensitivity one LOS absorption (QQ07) is detected, see Figs. 2 and 4 , and Table 3 . With a velocity limit of $5000 \mathrm{~km} / \mathrm{s}$, we find another case of LOS absorption, reported in Figs. 2, 4 and Table 3, with $\Delta v_{\text {rest }} \sim 2000 \mathrm{~km} / \mathrm{s}$, to be compared to the one case of Farina et al. (2014).

For the paucity of LOS with respect to transverse absorptions Farina et al. (2014) suggest as a possible explanation that the LOS absorbing clouds are heated at a temperature such that MgII is practically absent, but this is not the case in the transverse direction. This implies an anisotropy of the continuum emission. This picture was originally proposed 
by Prochaska et al. (2013) for the Ly $\alpha$ LOS absorptions. Our current dataset is still too small to further elaborate on this proposal. Since the distribution of the gas in the external regions is patchy, a sound statistical approach is required. A larger dataset of high quality and homogeneous spectra of quasar projected pairs would be able to draw firm conclusions on this intriguing scenario.

Funding Note Open access funding provided by Istituto Nazionale di Astrofisica within the CRUI-CARE Agreement.

Publisher's Note Springer Nature remains neutral with regard to jurisdictional claims in published maps and institutional affiliations.

Open Access This article is licensed under a Creative Commons Attribution 4.0 International License, which permits use, sharing, adaptation, distribution and reproduction in any medium or format, as long as you give appropriate credit to the original author(s) and the source, provide a link to the Creative Commons licence, and indicate if changes were made. The images or other third party material in this article are included in the article's Creative Commons licence, unless indicated otherwise in a credit line to the material. If material is not included in the article's Creative Commons licence and your intended use is not permitted by statutory regulation or exceeds the permitted use, you will need to obtain permission directly from the copyright holder. To view a copy of this licence, visit http://creativecommons.org/licenses/by/4.0/.

\section{References}

Cepa, J., Aguiar-Gonzalez, M., Bland-Hawthorn, J., Castaneda, H., Cobos, F.J., Correa, S., Espejo, C., et al.: Proc. SPIE 4841, 1739 (2003)

Farina, E.P., Falomo, R., Decarli, R., Treves, A., Kotilainen, J.K.: Mon. Not. R. Astron. Soc. 429, 1267 (2013)

Farina, E.P., Falomo, R., Scarpa, R., Decarli, R., Treves, A., Kotilainen, J.K.: Mon. Not. R. Astron. Soc. 441, 886 (2014)

Fielding, D., Quataert, E., McCourt, M., Thompson, T.A.: Mon. Not. R. Astron. Soc. 466, 3810 (2017)

Flesch, E.W.: Publ. Astron. Soc. Aust. 32, e010 (2015)

Flesch, E.W.: arXiv:1912.05614 (2019)

Gehrels, N.: Astrophys. J. 303, 336 (1986)

Hennawi, J.F., et al.: Astrophys. J. 651, 61 (2006)

Johnson, S.D., Chen, H.-W., Mulchaey, J.S.: Mon. Not. R. Astron. Soc. 452, 2553 (2015)

Paiano, S., Landoni, M., Falomo, R., Treves, A., Scarpa, R., Righi, C.: Astrophys. J. 837, 144 (2017)

Peek, J.E.G., Ménard, B., Corrales, L.: Astrophys. J. 813, 7 (2015)

Prochaska, J.X., et al.: Astrophys. J. 776, 136 (2013)

Oppenheimer, B.D., Davé, R., Kereš, D., Fardal, M., Katz, N., Kollmeier, J.A., Weinberg, D.H.: Mon. Not. R. Astron. Soc. 406, 2325 (2010)

Sharma, M., Nath, B.B., Chand, H.: Mon. Not. R. Astron. Soc. 431, L93 (2013)

Tumlinson, J., Peeples, M.S., Werk, J.K.: Annu. Rev. Astron. Astrophys. 55, 389 (2017)

Telford, O.G., Werk, J.K., Dalcanton, J.J., Williams, B.F.: Astrophys, J. 877, 120 (2019) 